\section{Unilateral linear syringoma in a Japanese female: dermoscopic differentiation from lichen planus linearis}

\author{
Yuka Hayashi, ${ }^{1}$ Masaru Tanaka, ${ }^{1}$ \\ Shizuka Nakajima, ${ }^{1}$ Masahiko Ozeki, ${ }^{1}$ \\ Takayuki Inoue, ${ }^{1}$ Sumiko Ishizaki, ${ }^{1}$ \\ Mariko Fujibayashi ${ }^{2}$ \\ Departments of ${ }^{1}$ Dermatology and \\ 2Pathology, Tokyo Women's Medical \\ University Medical Center East, Tokyo, \\ Japan
}

\begin{abstract}
A dermoscopy of linear unilateral syringoma has never been described in the English literature. The authors report the case to elucidate the dermoscopic features of syringoma. Clinical, dermoscopic and pathological features of a 38-year-old Japanese female case of linear unilateral syringoma on the left upper arm were described and compared with those of lichen planus linearis. Although the clinical appearance of linearly arranged dusky erythemas in the present case was quite similar to that of lichen planus linearis, dermoscopy did not show Wickham's striae but rather homogeneous light brown pigmentation with multifocal whitish areas and a delicate pigment network at the periphery. Dermoscopy might be helpful to differentiate linear unilateral syringoma from lichen planus linearis.
\end{abstract}

\section{Case Report}

A 38-year-old Japanese woman was referred to our department with an eight-year history of erythematous eruptions on the left upper arm. The patient denied a history of any medications or systemic disorders such as diabetes mellitus. She had no remarkable family history. A physical examination revealed an interrupted band composed of dusky erythemata on the left upper arm. The bands were small, nonscaling, asymptomatic erythemas, up to $5 \mathrm{~mm}$ in size (Figure 1A). A clinical diagnosis of lichen planus linearis was suspected.

A dermoscopic examination demonstrated a homogeneous light brownish area and a partial delicate, light brown pigment network at the periphery. Multifocal hypopigmentations were also noted in the lesions (Figure 1B). There were no obvious whitish pink arboriform or reticular structures corresponding to Wickham's striae. Although the dermoscopic findings were not typical, lichen planus linearis or lichen striatus was suspected, and a biopsy from the left upper arm was performed.

Histopathological examination of the hematoxylin and eosin-stained specimens disclosed the following (Figure 1C). The epidermis showed a slight thickening of the rete ridges with basal melanosis. In the upper dermis, numerous small ducts and islands of epithelium were embedded in a fibrous stroma. The duct walls were lined by two rows of epithelial cells. Clear cells were not observed. The lumina of the ducts contained amorphous material. Some of the ducts had small comma-like tails of epithelial cells, giving them the appearance of tadpoles. The clinico-pathological features suggested the diagnosis of unilateral linear syringomata.
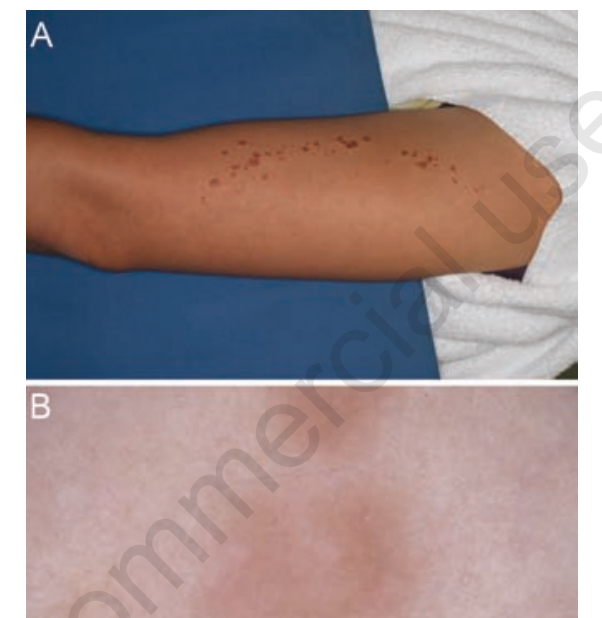

\section{Discussion}

Syringoma is a benign adnexal tumor arising from the ducts of eccrine sweat glands. It occurs predominantly in women at puberty or later in life. They are skin-coloured or light brown, small papules, usually 1 to $3 \mathrm{~mm}$ in diameter. In many patients, the lesions are limited to the lower eyelids and cheeks. Rarely the lesions of syringoma show a unilateral, linear arrangement. ${ }^{1}$ Linear syringomata usually present clinically quite similar to ordinary syringoma. However, the papules in the present case were larger than the usual size. Because of their common of linear arrangement, lichen striatus, lichen planus linearis, nevus comedonicus and nevoid basal cell epithelioma should occasionally be included in the differential diagnoses. ${ }^{1}$ Lichen planus linearis was especially suspected in our case. Therefore, we compared and evaluated examination of syringoma generally demonstrates homogeneous whitish or brownish areas and a partial delicate pigment network. ${ }^{2}$ The pigment network is considered one of the most specific parameters of melanocytic proliferation, and it correlates with the presence of melanin within the basal layer of the epidermis. The pigment network was also observed in our case, but it was delicate and very faint light brown in color, suggesting basal melanosis alone. A similar delicate, light brown pigment network is also seen in dermatofibroma. White areas on der- 
moscopy might be related to fibrous stroma surrounding the tumor nests. We speculated that fibrotic stroma in syringoma might cause epidermal thickening and basal melanosis, which would correspond to a delicate pigment network, as often shown in dermatofibroma. Dermoscopic features of lichen planus usually show white-pink linear arboriform structures, corresponding to Wickham's striae. White-pink structures would correspond to a band of lymphocytic infiltration at the upper dermis and saw-edged acanthosis together with orthokeratosis and hypergranulosis. These features that suggest lichen planus were not observed in our case. The linear distribution of the lesions may result from exogenous or lines invokes mosaicism, in which genetically distinct cell populations are produced by postzygotic mutations. ${ }^{3}$ Creamer et al. reported that the pathogenesis of syringoma is unclear; however, familial and Down's syndrome associations suggested the involvement of hereditary factors. ${ }^{3}$ Dermoscopy is considered as a helpful tool for increasing the diagnostic accuracy of histopathologic diagnoses of pigmented skin lesions. It is also useful when evaluating the less pigmented skin lesion by means of dermoscopy, as in our case. Syringoma should be included in the differential diagnoses when we encounter linearly arranged skin lesions for the pathological examination to yield a correct diagnosis.

\section{References}

1. Yung CW, Soltani K, Bernstein JE, Lorincz AL. Unilateral linear nevoidal syringoma. J Am Acad Dermatol 1981;4:412-6.

2. Ohara K. Syringoma. In; Saida T, et al., editors. Color Atlas Dermoscopy 1st. Tokyo: Kanehara-shuppan; 2003. pp. 175. (In Japanese).

3. Creamer D, Macdonald A, Griffiths WA Unilateral linear syringomata. A case report. Clin Exp Dermatol 1999;24:428-30. 